\title{
Comparing the hot and cold skies: INTEGRAL/BAT versus Planck
}

\section{J.B. Stephen}

INAF/IASF-Bologna,

Via P. Gobetti 101, 40129 Bologna, Italy

E-mail: stephen@iasfbo.inaf.it

\section{Bassani}

INAF/IASF-Bologna,

Via P. Gobetti 101, 40129 Bologna, Italy

E-mail: bassani@iasfbo.inaf.it

\section{A. Bazzano}

INAF/IASF-Roma,

Via Fosso del Cavaliere 100, 00133 Roma, Italy

E-mail: angela.bazzano@iasf-roma.inaf.it

\section{A.J. Bird}

School of Physics \& Astronomy

University of Southampton Highfield, Southampton, UK

E-mail:a.j.bird@soton.ac.uk

\begin{abstract}
A master catalogue of hard X-ray sources has been compiled from the various surveys performed with the INTEGRAL/IBIS and SWIFT/BAT telescopes. This represents the 'hot' view of the universe. A 'cold' view of the universe has recently been given in the form of the Planck Early Release Compact Source catalogue. We compare the two skies and find that only very few sources are common to both catalogues. These are generally active galaxies, mainly Blazars and Seyfert 2's. The latter are probably detected due to a mixture of the AGN and ongoing starburst activity.
\end{abstract}

The Extreme and Variable High Energy Sky,

Chia Laguna (Cagliari), Italy

September 19-23, 2011 


\section{Introduction}

We have used the published hard X-ray catalogues from INTEGRAL/IBIS and SWIFT/BAT, symbolising the hot sky and the Planck Early release point source catalogue, representing the cold sky and searched for sources common to both. Clearly one would expect to find very little in common between to such extreme examples of celestial emission. Nevertheless, should there be a certain commonality found, this could provide important information about the nature of these objects.

\section{The Catalogues}

The 'Master' Hard X-ray catalogue was compiled from 4 catalogues: two from the SWIFT/BAT telescope - the second Palermo 54 month [1]; and the 58 month survey [2] and two from the INTEGRAL/IBIS instrument - the $4^{\text {th }}$ Catalogue [3] and the 7-year all sky hard Xray survey [4]. Merging these four catalogues and removing obvious double entries leads to a list containing 1574 individual sources. The Planck data used was the $350 \mu \mathrm{m}(857 \mathrm{GHz})$ list from the Early Release Compact Source catalogue (ERCSC, [5]) containing 8988 entries. From a simple spatial correlation analysis we find that there are a total of 54 sources detected in both the hard-X and GHz frequencies. Of these there are 5 galactic objects (e.g. The Crab) and one cluster of galaxies. Table 1 lists the remaining 48 sources from which it is apparent that they are dominated by active galaxies, in particular Seyfert 2s.

\section{Source Classification}

The source type in table 1 has been obtained in general from NED/Veron-Veron $13^{\text {th }}$ edition [6], however this was not possible for all objects as either the information was not available (rows in italics) or ambiguous/partial (rows in bold font). For example, Gonçalves et al. [7] classified the optical spectrum of NGC 4102 as composite, concluding that the nucleus is dominated by starburst emission, although a weak Type 2 Seyfert component is also present; the AGN component has recently been confirmed by a Suzaku observation of the source [8]. Further, IC 2461 has recently been observed within our optical follow-up program of high energy sources and classified as a Seyfert 2 galaxy (see fig 1. and [9]) . 


\begin{tabular}{|c|c|c|c|c|c|}
\hline Hard X Name & Planck Name & GHz Flux & X-rav Flux & $\mathbf{Z}$ & Type \\
\hline 3C454.3 & G086.11-3 & 18340.7 & 131.8 & 0.8590 & Blazar \\
\hline $3 \mathrm{C} 273$ & G289.96+6 & 4065.28 & 430.84 & 0.1583 & Blazar \\
\hline 3C 279 & G305.11+5 & 3333.66 & 34.82 & 0.5362 & Blazar \\
\hline PKS 0537-441 & G250.08-3 & 3057.01 & 14.16 & 0.8940 & Blazar \\
\hline NGC 4102 & G138.07+6 & 9084.47 & 28.55 & 0.0028 & Liner \\
\hline NGC 4593 & G297.47+5 & 3244.15 & 88.68 & 0.0090 & S1 \\
\hline NGC 235A & G093.84-8 & 3025.72 & 47.8 & 0.0222 & S1 \\
\hline NGC 7469 & G083.09-4 & 5438.24 & 68.69 & 0.0163 & S1.2 \\
\hline NGC 7214 & G022.23-5 & 2428.57 & 64.97 & 0.0231 & S1.2 \\
\hline NGC 4151 & G155.06+7 & 2625.54 & 533.09 & 0.0033 & S1.5 \\
\hline NGC6814 & G029.34-1 & 7747.16 & 75.29 & 0.0052 & S1.5 \\
\hline NGC 1566 & G264.30-4 & 16870.6 & 20.08 & 0.0050 & S1.5 \\
\hline NGC 3227 & G216.98+5 & 5879.35 & 112.78 & 0.0039 & S1.5 \\
\hline NGC 7213 & G349.58-5 & 3879.45 & 44.3 & 0.0058 & S1.5 \\
\hline NGC 4051 & G148.87+7 & 10500.1 & 37.62 & 0.0023 & S1.5 \\
\hline IGRJ12131+0700 & G276.81+6 & 1839.95 & 14.44 & 0.2095 & S1.5-1.8 \\
\hline NGC 1365 & G237.95-5 & 43160 & 64.47 & 0.0055 & S1.8 \\
\hline NGC 3786 & G191.54+7 & 3018.31 & 20.49 & 0.0089 & S1.8 \\
\hline NGC 4395 & G162.11+8 & 6199.53 & 26.08 & 0.0011 & S1.8 \\
\hline NGC 2992 & G249.71+2 & 3597.66 & 30.13 & 0.0077 & S1.9 \\
\hline NGC 5674 & G355.89+5 & 1357.17 & 13.61 & 0.0249 & S1.9 \\
\hline NGC 5290 & G089.27+7 & 2500.52 & 19.14 & 0.0086 & S1.9 \\
\hline NGC 5728 & G337.32+3 & 4939.8 & 90.9 & 0.0094 & S1.9 \\
\hline NGC 4138 & G147.30+7 & 2717.09 & 30.67 & 0.0030 & S1.9 \\
\hline M58 & G290.41+7 & 8989.44 & 10.41 & 0.0051 & S1.9 \\
\hline UGC 07064 & G188.54+7 & 1071.49 & 13.64 & 0.0250 & S1.9 \\
\hline IC 2461 & $G 186.28+4$ & 1146.39 & 20.73 & 0.0075 & $S 2$ \\
\hline CEN A & G309.51+1 & 115319 & 1379.67 & 0.0018 & S2 \\
\hline NGC 4388 & G279.12+7 & 4857.95 & 275.78 & 0.0084 & S2 \\
\hline NGC 4945 & G305.27+1 & 297415 & 300.97 & 0.0019 & S2 \\
\hline NGC 6300 & G328.49-1 & 13484.2 & 96.99 & 0.0037 & S2 \\
\hline NGC 5643 & G321.44+1 & 13688 & 18.33 & 0.0040 & S2 \\
\hline NGC 4258 & G138.33+6 & 25691 & 23.92 & 0.0015 & S2 \\
\hline NGC 4939 & G308.09+5 & 4561.28 & 25.43 & 0.0104 & S2 \\
\hline NGC 6240 & G020.72+2 & 3469.74 & 66.96 & 0.0245 & S2 \\
\hline 4C55.19 & G157.80+4 & 21735.7 & 31.1 & 0.0037 & S2 \\
\hline MCG-01-05-047 & G157.36-6 & 2504.66 & 22.33 & 0.0172 & S2 \\
\hline NGC 5899 & G069.38+5 & 4585.51 & 20.95 & 0.0085 & S2 \\
\hline NGC 612 & G261.65-7 & 1354.66 & 57.11 & 0.0298 & S2 \\
\hline NGC 4941 & G308.79+5 & 2922.98 & 19.41 & 0.0037 & S2 \\
\hline NGC 2655 & G134.91+3 & 2969 & 14.05 & 0.0047 & S2 \\
\hline NGC 1068 & G172.09-5 & 48923.7 & 33.75 & 0.0038 & S2 \\
\hline NGC 7582 & G348.07-6 & 14057.4 & 80.95 & 0.0053 & S2 \\
\hline NGC 835 & G173.96-6 & 5351 & 17.72 & 0.0136 & S2 \\
\hline IC1663 & G262.57-8 & 1847.79 & 15.32 & 0.0361 & S2 \\
\hline NGC 2712 & $G 175.64+41$ & 2257.52 & 10 & 0.0061 & S2? \\
\hline NGC4500 & G128.08+58 & 966.87 & 8.69 & 0.0104 & SB(S2) \\
\hline M82 & G141.40+4 & 156993 & 5.96 & 0.0007 & SB \\
\hline
\end{tabular}

Table 1. The 48 extragalactic sources in common between the Planck ERCSC and the Hard X-ray 'Master' catalogues. The Planck flux is in units of $10^{-26} \mathrm{ergs} \mathrm{s}^{-1} \mathrm{~cm}^{-2}$ $\mathrm{Hz}^{-1}$ while the X-ray flux is in units of $10^{-12}$ ergs s $\mathrm{cm}^{-2}$. 
For two other sources (NGC 4500 and NGC 2712) SWIFT-XRT data can help in understanding the nature of the galaxy: both objects show absorption in excess of the galactic value (41 and $4 \times 10^{22} \mathrm{~cm}^{-2}$ respectively) and an unabsorbed 2-10 keV flux of 4.6 and $3.5 \times 10^{-12}$ $\mathrm{erg} / \mathrm{cm}^{2}$ sec respectively, which at the sources' redshifts indicate soft X-ray luminosities of $10.9 \times 10^{41} \mathrm{erg} / \mathrm{sec}$ for NGC 4500 and $2.8 \times 10^{41} \mathrm{erg} / \mathrm{sec}$ for NGC 2712. Combined with the hard (14-195 keV) X-ray luminosities of 20 and $810^{41}$ the observed values suggest an obscured AGN nature for both of these sources. Assuming that both these galaxies are type 2 objects from the detection of intrinsic absorption we conclude that the associations reported here consists of only 1 pure starburst galaxy and 47 AGN: 11 of type 1, 32 of type 2 and 4 blazars of the Low Synchrotron Peak type (i.e. with a first peak below $10^{14} \mathrm{~Hz}$ ).

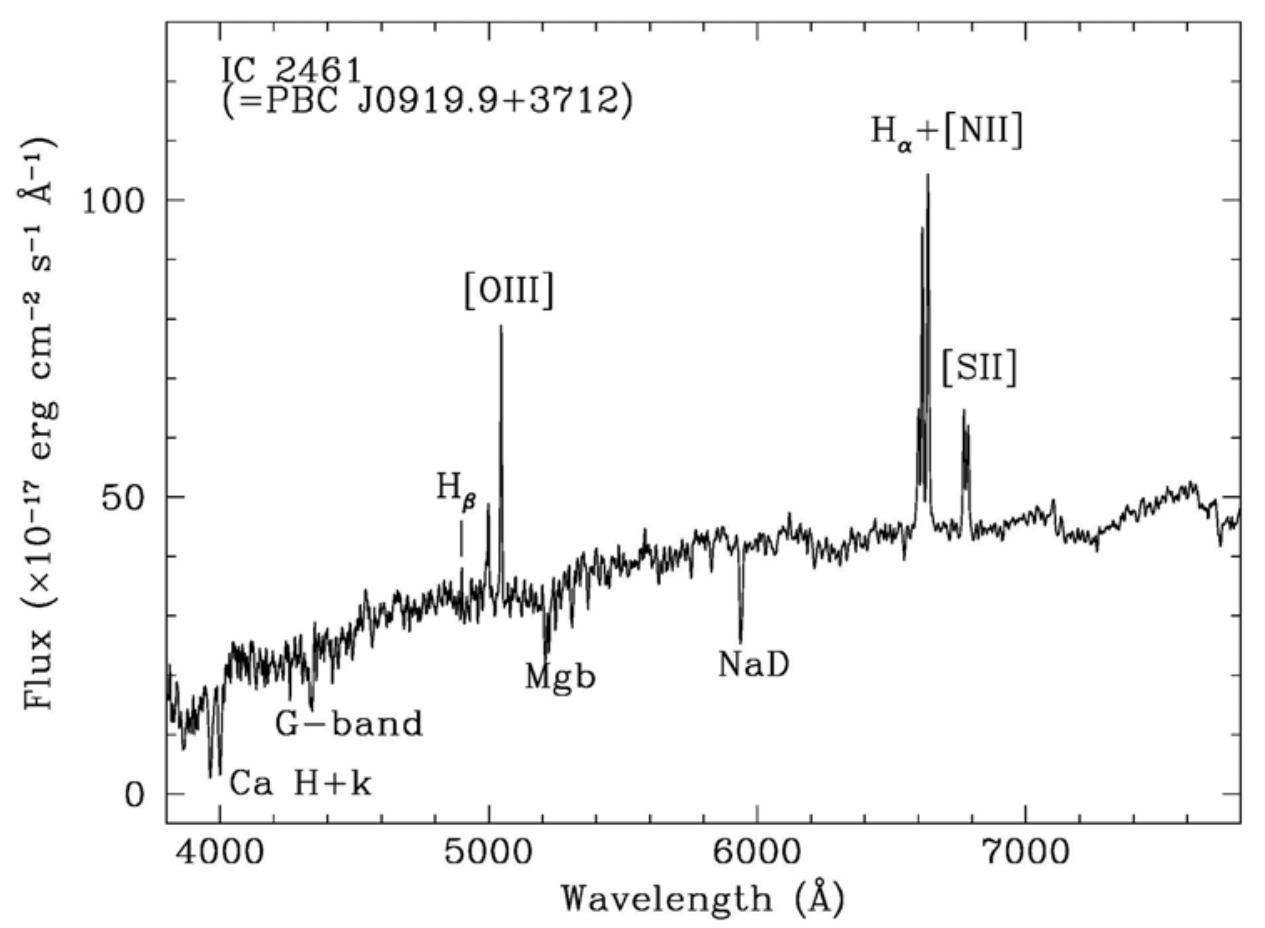

Fig 1. The optical spectrum of IC 2641 demonstrating the lack of broad line emission features leading to its classification as a type 2 Seyfert. 


\section{Discussion}

It is interesting that, while there is a very small number of objects detected both by Planck and the Hard X-ray telescopes, the vast majority are extra-galactic in origin. For these sources the Hard X-ray observations probe the AGN phenomenon, while the $\mathrm{GHz}$ measurements track not only the nuclear component (radio loud in blazars and quiet in Seyferts), but also the presence of star formation. Therefore it is likely that the Seyfert sample will be dominated by composite starburst/AGN galaxies. Indeed, within table 1 we find many such objects e.g. NGC 6240 is one of the best examples of a galaxy hosting a luminous starburst simultaneously with AGN activity, while NGC 4102 is also a composite dominated by star formation. Other examples include NGC 1068, NGC 4388, NGC 4945 and NGC 7469. The fact that the vast majority of Seyfert galaxies in the sample are of type 2 suggests that star formation is more prevalent in this class, with it being suggested that up to 50\% of all Seyfert 2s exhibit also circumnuclear starbursts, although this is still a matter of debate.

Composite starburst/AGN galaxies are of interest due to the still unclear relationship, if any, between the two phenomena, the distribution in fluxes between them (and so their relative contribution to the cosmic X-ray background) and the great difficulty in identifying composite galaxies. It would appear that Planck measurements over a wide operational range from 350 $10000 \mu \mathrm{m}$ will provide a new tool with which to study these objects in a relatively unexplored region of the spectrum. Combining these 'cold' and 'hot' observations will both increase the numbers of known composite galaxies and help untangle the two components.

\section{References}

[1] Cusumano, G. et al (2010) A\&A 52464

[2] B. Baumgartner et al (2010) and http://heasarc.gsfc.nasa.gov/docs/swift/results/bs58month

[3] Bird, A.J. et al (2010) ApJS 1861

[4] Krivonos, R. et al (2010) A\&A 52361 and http://hea.iki.rssi.ru/integral/survey/catalog.php?status=current

[5] Ade, P.A.R. et al arXiv 1101.2041v2

[6] Veron-Cetty \& Veron (2010) A\&A 518,10

[7] Gonçalves, A.C. et al (1999) A\&AS 135437

[8] Gonzales-Martin et al (2011) A\&A 527142

[9] Parisi, P. private communication 